

\title{
On the determination of eigenfrequencies for nanometer-size objects
}

\author{
V. A. Eremeyev, E. A. Ivanova, N. F. Morozov, A. N. Solovev
}

\section{To cite this version:}

V. A. Eremeyev, E. A. Ivanova, N. F. Morozov, A. N. Solovev. On the determination of eigenfrequencies for nanometer-size objects. Doklady Physics, 2006, 51 (2), pp.93-97. hal-00830676

\section{HAL Id: hal-00830676 https://hal.science/hal-00830676}

Submitted on 5 Jun 2013

HAL is a multi-disciplinary open access archive for the deposit and dissemination of scientific research documents, whether they are published or not. The documents may come from teaching and research institutions in France or abroad, or from public or private research centers.
L'archive ouverte pluridisciplinaire HAL, est destinée au dépôt et à la diffusion de documents scientifiques de niveau recherche, publiés ou non, émanant des établissements d'enseignement et de recherche français ou étrangers, des laboratoires publics ou privés. 


\title{
On the Determination of Eigenfrequencies for Nanometer-Size Objects
}

\author{
V. A. Eremeyev ${ }^{1}$, E. A. Ivanova ${ }^{2}$, Academician N. F. Morozov ${ }^{3}$, and A. N. Solov'ev ${ }^{4}$
}

\section{INTRODUCTION}

At present, the problem of the experimental determination of mechanical characteristics for nanometersize objects (nanoobjects) is urgent. One of the most efficient methods for the determination of elastic-moduli is based on the measurement of the eigenfrequencies of objects under study. However, the measurement of nanoobject frequencies, in particular, on the basis of optical methods turns out to be problematic [1]. The main, though not exclusive, limitation upon the application of optical methods is the fact that the laser-beam cross section is not a point but a spot whose diameter is on the order of the light wavelength. Thus, if the size of an object under investigation is smaller than the diameter of the laser beam, the measurement results are meaningless. Meanwhile, the use of optical methods makes it possible to measure eigenfrequencies for systems containing microscopic-size substrates (microsubstrates) and a regular structure of identical nanoobjects. As a result, two problems arise that lie at the interface between mechanics and experimental physics. The first problem consists in the determination of the elastic moduli of nanoobjects under conditions in which the frequencies of a combined system of a microsubstrate and nanoobjects and of isolatedmicrosubstrate elastic properties are known. The sec-

\footnotetext{
${ }^{1}$ South Scientific Center, Russian Academy of Sciences, and Rostov State University,

ul. Zorge 5, Rostov-on-Don, 344090 Russia

${ }^{2}$ St. Petersburg State Polytechnical University, Polytekhnicheskaya ul. 29, St. Petersburg, 195251 Russia

3 St. Petersburg State University, Bibliotechnaya pl. 2, Stary̆ Peterhof, 198904 Russia

${ }^{4}$ Donskoŭ State Technical University. ul. Zorge 5, Rostov-on-Don, 344090 Russia

e-mail:eremevev@math.rsu.nu;

ivanova@E15063.spb.edu; morozov@NM1016.spb.edu; soloviev@math.rsu.ru
}

ond problem is associated with the possibility of isolating the nanoobject eigenfrequencies in the microsubstrate-nanoobject system. The successful solution of both problems directly depends on the conditions under which the experiment is performed. Thus, the role of mechanics consists not only in the interpretation of experimental data but also in the development of the theoretical foundations of experimental approaches and of recommendations related to various mechanical aspects of the problem.

In this paper, we propose a method for the determination of the eigenfrequencies of certain nanostructures (e.g., nanotubes and nanocrystals). The method is based on measuring eigenfrequencies of an "extended system" consisting of a highly oriented array (lattice) of identical nanotubes or nanocrystals located on a substrate. Structures of this kind can be obtained as a result of processes of self-organized growth $[2,3]$. As a rule, the sizes of these nanoobjects in the array are approximately the same, which makes it possible to use the macroscopic size of this array to study nanoobject properties by the determination of first eigenfrequencies for either nanotube-lattice or nanocrystal-substrate systems. We show that, using the found spectrum of the extended (lattice-substrate) system and the substrate spectrum, it is possible to determine eigenfrequencies of the unique nanoobject. This allows us to determine nanoobject eigenfrequencies on the basis of data obtained for extended systems.

The fundamental problem that arises in measuring frequencies of the objects under study, which are fixed at an elastic substrate, is the oscillation-eigenfrequency redistribution in the system consisting of the objects and the substrate between eigenfrequencies of each of them taken separately. This method is well known in mechanics [4]. In this case, the character of the spectrum shift substantially depends on the relation between the parameters of both the object under study and the substrate. Meanwhile, it is well known that, in distributed-parameter systems consisting of several bodies, a phenomenon (anti-resonance) exists that con- 
sists of the dynamically damping vibrations of one body at the partial frequency of the other body. This phenomenon is the basis for the method of measuring nanoobject eigenfrequencies by means of an atomicforce microscope. Below, we show that, in systems of a highly oriented array of identical nanotubes or nanocrystals grown on a substrate, the anti-resonance phenomenon also takes place and can be applied to the isolation of nanoobject eigenfrequencies from the extended-system spectrum. It is unlikely that the analytical study of eigenmodes of such a system can be performed in the framework of the three-dimensional elasticity theory. Therefore, as the first stage of the investigation, we consider the rod model of an extended system, which consists of a horizontal rod modeling a substrate and vertical rods modeling nanoobjects. For the rod model, the analysis of eigenmodes for a nanocrystal system is carried out, and the possibility of the isolation of the nanoobject spectrum from the extended-system spectrum is proved. At the second stage, the dynamics of a concrete mechanical system is numerically analyzed. In the framework of the plane problem of the elasticity theory, calculations of the lattice frequencies are carried out for the lattice of zinc oxide microcrystals and nanocrystals on the sapphire substrate. The calculation results also demonstrate the possibility of isolating the nanoobject spectrum from that of the extended system.

\section{THE ANALYTICAL STUDY OF THE MODEL PROBLEM}

We consider the rod model of an extended system consisting of both a horizontal rod of length $L$ and $N$ vertical rods each of length $H$ fixed at their lower ends to the horizontal rod and located at the same distance $l$ from each other so that $L=(N+1) l$. Upper ends of the vertical rods are free, whereas the ends of the horizontal rod also are rigidly fixed. We introduce the following notation: $C$ and $D$ are flexural rigidities of the horizontal and vertical rods, respectively; $\rho_{1}, \rho_{2}$ are the linear mass densities; $u, \varphi=u^{\prime}$ are the vertical displacement and the rotation angle of the horizontal rod; $\mathrm{v}_{n}, w_{n}$, $\psi_{n}=-V_{n}$ are the horizontal and vertical displacements and the rotation angle for the $n$th vertical $\operatorname{rod} ; T, M=$ $C u^{\prime \prime}$ is the transverse force and the bending moment in the horizontal-rod cross section; $N_{n}=-D v_{n}^{\prime \prime \prime}$, and $F_{n}$, $L_{n}=-D V_{n}^{\prime \prime}$ are the transverse and longitudinal forces and the bending moment in the cross section of the $n$th vertical rod.

The equations of motion for the vertical rods are of the form

$$
D V_{n}^{\mathrm{IV}}+\rho_{2} \ddot{V}_{n}=0, \quad w_{n}^{\prime}=0, \quad F_{n}^{\prime}=\rho_{2} \ddot{w}_{n}
$$

The equations of motion for the horizontal rods can be written out as

$$
\begin{gathered}
T^{\prime}+\left.\sum_{n=1}^{N} F_{n}\right|_{y=0} \delta(x-n l)=\rho_{1} \ddot{u}, \\
M^{\prime}+T+\left.\sum_{n=1}^{N} L_{n}\right|_{y=0} \delta(x-n l)=0 .
\end{gathered}
$$

The vertical rods are rigidly fixed at the horizontal one so that the conjugation kinematic conditions are valid:

$$
\left.V_{n}\right|_{y=0}=0,\left.\quad w_{n}\right|_{y=0}=\left.u\right|_{x=n l},\left.\quad \psi_{n}\right|_{y=0}=\left.\varphi\right|_{x=n l} .
$$

The boundary conditions for the entire system are formulated as

$$
\begin{aligned}
\left.u\right|_{x=0}=0, & \left.\varphi\right|_{x=0}=0,\left.\quad u\right|_{x=L}=0, \\
& \left.\varphi\right|_{x=L}=0, \\
\left.N_{n}\right|_{y=H}=0, & \left.F_{n}\right|_{y=H}=0,\left.\quad L_{n}\right|_{y=H}=0 .
\end{aligned}
$$

The solution to Eqs. (1) describing the motion of the vertical rods in combination with boundary conditions (4) at free ends of the vertical rods makes it possible to link forces and displacements in lower points of the rods:

$$
\begin{aligned}
\left.F_{n}\right|_{y=0} & =-\left.\rho_{2} H \ddot{w}_{n}\right|_{y=0}, \\
\left.L_{n}\right|_{y=0} & =\left.\frac{D \mu}{g(\mu H)} \psi_{n}\right|_{y=0},
\end{aligned}
$$

where the parameter $g(\mu H)$ is of the form

$$
g(\mu H)=\frac{1+\cos (\mu H) \cosh (\mu H)}{\sin (\mu H) \cosh (\mu H)-\cos (\mu H) \sinh (\mu H)} .
$$

Removing the transverse force $T$ from Eq. (2) and taking into account the elasticity relation $M=C u$ " and relationships (3), (5), we reduce the set of Eqs. (2) to the unique differential equation

$$
\begin{gathered}
C u^{\mathrm{IV}}+\rho_{1} \ddot{u}=-\sum_{n=1}^{N}\left[\rho_{2} H \ddot{u} \delta(x-n l)\right. \\
\left.+\frac{D \mu}{g(\mu H)} u^{\prime} \delta^{\prime}(x-n l)\right] .
\end{gathered}
$$

If the number of vertical rods is sufficiently large, we can consider them to be continuously distributed along the horizontal-rod length. Upon averaging the right- 
hand side of Eq. (6), we simplify the mathematical formulation of the problem and reduce it to the equation

$$
u^{\mathrm{IV}}-\frac{N D \mu}{C g(\mu H) L} u^{\prime \prime}+\frac{\rho_{1}}{C}\left(1+N \frac{\rho_{2} H}{\rho_{1} L}\right) \ddot{u}=0 .
$$

The analysis of the above-formulated problem shows the existence of two groups of solutions. The first one corresponds to the situation when the vertical rods move as a cantilever beams. The eigenfrequencies of system vibrations are determined by the equation

$$
1+\cos (\mu H) \cosh (\mu H)=0, \quad \mu=\sqrt[4]{\frac{\rho_{2}}{D}} \sqrt{\omega} .
$$

In this case, the vibration amplitudes for the horizontal rod are small compared to those of the vertical rods. The second group of solutions corresponds to the situation when the system vibrates at frequencies close to those for the system consisting of one horizontal rod. Here, the vibration amplitudes for the vertical rods are small compared to those of the horizontal rod.

The comparison of quantities $\lambda L$ and $\mu H$ ( here, $\lambda=$ $\left.\sqrt[4]{\frac{\rho_{1}}{C} \sqrt{\omega}}\right)$ allows us to determine the mutual position of both the substrate and the nanoobject spectra. The first eigenfrequencies of the substrate and of the nanoobjects correspond to the values $\lambda L \sim 1$ and $\mu H \sim 1$, respectively. If $\frac{\mu H}{\lambda L} \ll 1$, then the first eigenfrequencies of the nanoobjects are significantly lower than those of the substrate. If $\frac{\mu H}{\lambda L} \gg 1$, then the first eigenfrequencies of the nanoobjects significantly exceed that of the substrate. The following estimate is true:

$$
\frac{\mu H}{\lambda L} \sim\left(\frac{h_{1}}{h_{2}}\right)^{\frac{1}{2}} \frac{H}{L},
$$

where $h_{1}$ and $h_{2}$ are the characteristic sizes of cross sections for the horizontal and the vertical rods, respectively.

Thus, in the case of modeling the extended system and the substrate in the framework of rod mechanics, the isolation from the extended-system spectrum of eigenfrequencies corresponding to the frequency of a unique nanoobject is possible.

\section{NUMERICAL ANALYSIS OF THE DYNAMICS OF A REAL SYSTEM}

As an example of a real nanostructure, we consider the problem of the isolation of eigenfrequencies for a

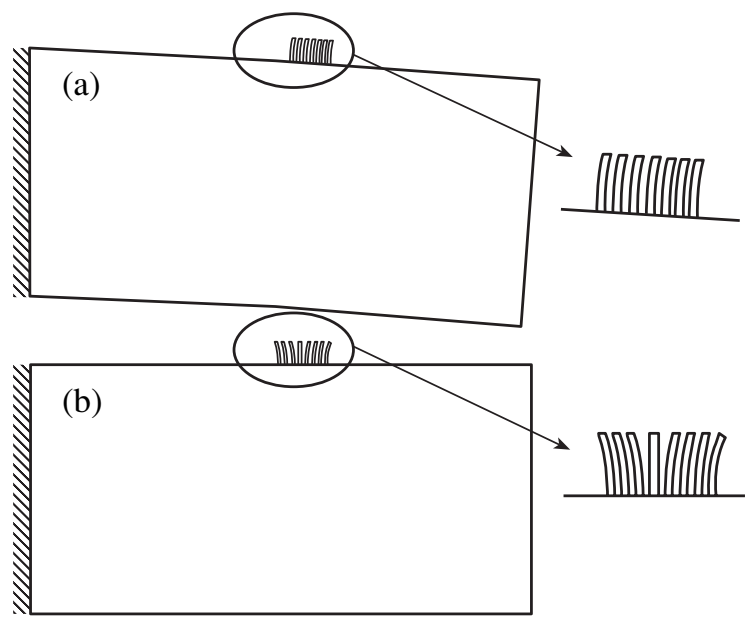

Fig. 1. Eigenfrequencies (a) of the substrate and (b) localized in the nanocrystal lattice.

lattice of zinc oxide ( $\mathrm{ZnO}$ ) microcrystals and nanocrystals. $\mathrm{ZnO}$ single crystals are piezoelectrics. By virtue of their excellent optical and mechanical properties, they have far-reaching prospects for application in nanoelectronics, nanophotonics, and nanomechanics. In particular, they are used for constructing UV lasers, sensors of chemical and biological substances, solar batteries, UV photodetectors, and other devices. $\mathrm{ZnO}$ single nanocrystals are synthesized by different methods, e.g., by thermal evaporation, chemical deposition from the gas phase, by pulsed laser spraying, etc. The height and diameter of the nanocrystals attain $1.5-3 \mu \mathrm{m}$ and $30-$ $100 \mathrm{~nm}$, respectively. Microcrystals are $20-100 \mu \mathrm{m}$ in height and 1.0-3.0 $\mu \mathrm{m}$ in diameter. From the standpoint of continuum mechanics, the nanocrystal lattice on a substrate is a compound piezoelectric solid. The basic electric-elasticity equations in the electrostatic approximation and in the absence of mass forces are of the form [5-7]

$$
\begin{gathered}
\rho \ddot{\mathbf{u}}=\nabla \cdot \boldsymbol{\sigma}, \quad \nabla \cdot \mathbf{D}=0, \\
\boldsymbol{\sigma}=\mathbf{C} \cdot \boldsymbol{\varepsilon}-\mathbf{e} \cdot \mathbf{E}, \quad \mathbf{D}=\mathbf{e} \cdot \boldsymbol{\varepsilon}+\boldsymbol{\epsilon} \cdot \mathbf{E}, \\
\boldsymbol{\varepsilon}=\frac{1}{2}\left(\nabla \mathbf{u}+\nabla \mathbf{u}^{T}\right), \quad \mathbf{E}=\nabla \varphi .
\end{gathered}
$$

Here, $\mathbf{u}$ is the displacement vector; $\mathbf{E}$ is the vector of the electric-field strength, which is expressed in terms of the potential $\varphi ; \boldsymbol{\sigma}$ is the stress tensor; $\mathbf{D}$ is the electricinduction vector; $\boldsymbol{\varepsilon}$ is the strain tensor; $\nabla$ is the gradient operator; $\rho$ is the density; and $\mathbf{C}, \mathbf{e}$, and $\boldsymbol{\epsilon}$ are the rigidity matrix and the piezoelectric and dielectric constants, respectively. Below, we restrict our analysis to the case of a plane problem.

The model analysis of Eqs. (7)-(9) with the corresponding boundary conditions for the $\mathrm{ZnO}$ nanocrystals on the sapphire substrate was performed on the 


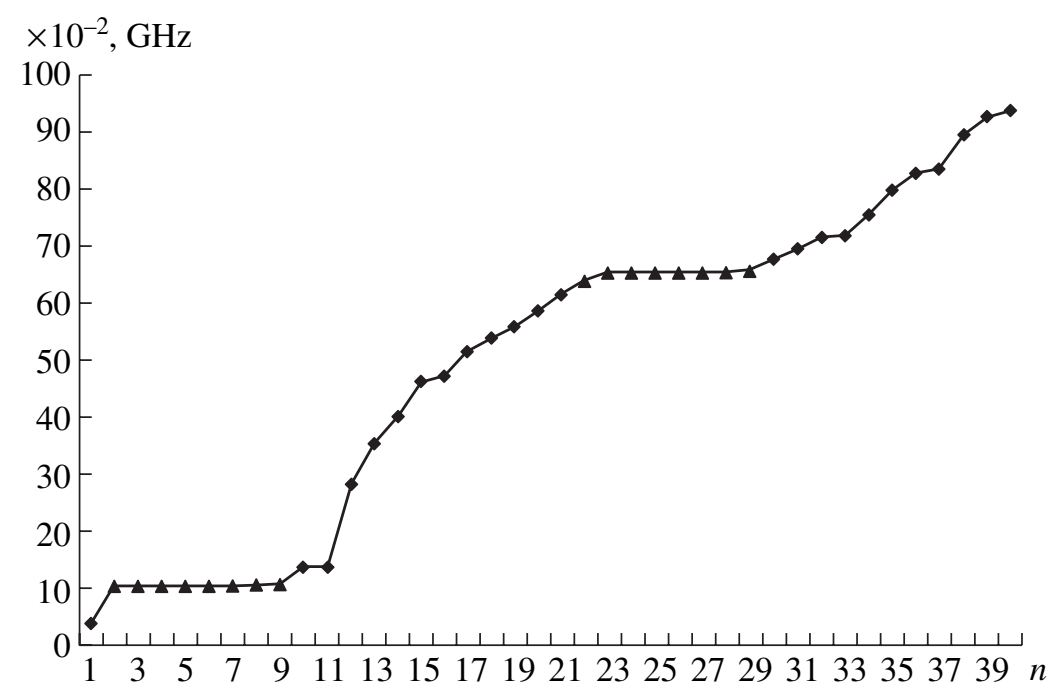

Fig. 2. Distribution of eigenfrequencies for the extended system. Triangles and rhombi correspond to the frequencies of the unique nanocrystal and of the substrate, respectively.

basis of the ACELAN finite-element program package [8-10]. The properties of both $\mathrm{ZnO}$ single crystals and the sapphire substrate were taken from [11].

In the numerical experiment, we have found eigenfrequencies of a cantilever fixed $\mathrm{ZnO}$ nanocrystal $1 \mu \mathrm{m}$ in height and $0.1 \mu \mathrm{m}$ thick. The first and second frequencies turned out to be 0.10797 and $0.67763 \mathrm{GHz}$, respectively, and correspond to bending vibrations. A sapphire single crystal of the rectangular shape $(10 \times$ $20 \mu \mathrm{m}$ ) and fixed at one lateral side was considered as a substrate model. The extended system was modeled by the above-described sapphire microcrystal with a sampling of eight identical nanocrystals located on the upper substrate side. The results of the model analysis

\section{Table}

\begin{tabular}{c|c}
\hline $\begin{array}{c}\text { Eigenfrequencies of the } \\
\text { extended system, } \mathrm{GHz}\end{array}$ & $\begin{array}{c}\text { Eigenfrequencies of the } \\
\text { substrate, } \mathrm{GHz}\end{array}$ \\
\hline 0.036494 & 0.036594 \\
0.103909 & - \\
0.103971 & - \\
0.104039 & - \\
0.104106 & - \\
0.104226 & - \\
0.104322 & - \\
0.104467 & - \\
0.104612 & - \\
0.134652 & 0.134973 \\
0.136246 & 0.136017 \\
0.280004 & 0.280137 \\
\hline
\end{tabular}

are presented in the table. Missed rows in the second column are associated with the absence of the corresponding eigenfrequencies for the substrate. Examples of the shapes of eigenmodes are shown in Fig. 1. Figure 1a corresponds to the first frequency in the table. The kinematic analysis of eigenmodes shapes corresponding to the consequent eight eigenfrequencies testifies to the fact that motions of the extended system are localized in the nanocrystalline "brush" (Fig. 1b). In this case, the shape of the nanocrystal vibrations corresponds to the first eigenfrequency of the unique nanocrystal. The vibration frequencies of the nanocrystal ensemble differ from that of a unique nanocrystal by less than $4 \%$. The analogous dynamic behavior of the extended system manifests itself in the vicinity of the second eigenfrequency of a unique nanocrystal. This is illustrated in Fig. 2, in which the plateaus of each plotted curve correspond to the eigenfrequencies of the unique nanocrystal. Here, triangles and rhombi denote frequencies corresponding to those of the unique nanocrystal and of the substrate, respectively.

The calculations have shown that the extended-system spectrum can be approximately represented as a combination of substrate eigenfrequencies and frequencies generated by a unique nanocrystal, which is quite consistent with the results of the rod-model analysis. Thus, the model proposed makes it possible to experimentally determine to a high accuracy the first eigenfrequencies of a unique nanoobject on the basis of spectra of the lattice-substrate system and of the unique substrate.

\section{ACKNOWLEDGMENTS}

This work was supported by the Russian Foundation for Basic Research, project nos. 03-07-90411 and 
05-01-00094 and by the President of Russian Federation, grant no. MD-3475.2005.1.

\section{REFERENCES}

1. R. H. Marshall, I. A. Sokolov, Y. N. Ning, et al., Meas. Sci. Technol. 7, 1683 (1996).

2. Springer Handbook of Nanotechnology, Ed. by B. Bhushan (Springer, Berlin, 2004).

3. Handbook of Nanoscience, Engineering and Technology, Ed. by W. A. Goddart, D. W. Brenner, S. E. Lyshevski, et al. (CRC, Boca Raton, 2003).

4. S. Gould, Variational Methods for Eigenvalue Problems; An Introduction to the Weinstein Method of Intermediate Problems (Oxford Univ. Press, London, 1966; Mir, Moscow, 1970).

5. G. Maugin, Continuum Mechanics of Electromagnetic Solids (North-Holland, Amsterdam, 1988; Mir, Moscow, 1991).
6. V. Z. Parton and B. A. Kudryavtsev, Electromagnetic Elasticity of Piezoelectric and Conducting Bodies (Nauka, Moscow, 1988) [in Russian].

7. W. Nowacki, Electromagnetic Effects in Solids (PWN, Warszawa, 1983 [in Polish]; Mir, Moscow, 1986 [in Russian]).

8. A. V. Belokon', V. A. Eremeyev, A. V. Nasedkin, and A. N. Solov'ev, Prikl. Mat. Mekh. 64 (3), 381 (2000).

9. O. N. Akopov, A. V. Belokon', K. A. Nadolin, et al., Mat. Model. 13 (2), 51 (2001).

10. K. E. Vasil'chenko, A. V. Nasedkin, and A. N. Solov'ev, Vychisl. Tekhnol. 10 (1), 10 (2005).

11. A. A. Blistanov, V. S. Bondarenko, N. V. Perelomova, et al., Acoustical Crystals. Handbook (Nauka, Moscow, 1982) [in Russian]. 